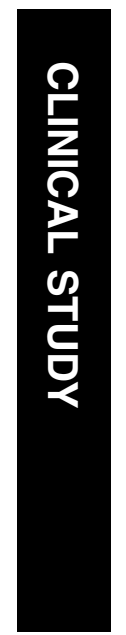

\title{
Malignant eyelid tumours in Taiwan
}

\author{
Abstract \\ Aims To describe the clinical characteristics \\ of patients with eyelid cancers in Taiwan. \\ Methods Between 1980 and 2000, 127 (58 \\ males and 69 females) patients (mean age 62.6; \\ range 10-91 years) with histologically \\ confirmed eyelid cancers were retrospectively \\ evaluated at the National Taiwan University \\ Hospital and Far Eastern Memorial Hospital in \\ Taiwan. Clinical data of all patients were \\ reviewed from medical records. The mean \\ follow-up period was 62.4 months (range \\ 3-240) for 113 patients. \\ Results The 127 eyelid cancers included 79 \\ basal cell carcinomas $(62.2 \%), 30$ sebaceous \\ gland carcinomas $(23.6 \%), 11$ squamous cell \\ carcinomas $(8.7 \%)$, five malignant melanomas \\ (3.9\%), one Kaposi's sarcoma $(0.8 \%)$, and one \\ metastatic cancer $(\mathbf{0 . 8 \%})$. Tumours developed \\ more commonly in the lower $\mathbf{3 7 . 0 \% )}$ than the \\ upper eyelid $(33.9 \%)$. The clinical accuracy in \\ predicting eyelid malignancy was $\mathbf{9 0 . 5 \%}$. \\ Primary treatment modality was mainly \\ surgical excision. The recurrence, \\ metastasis, and mortality rates at 5 years \\ were $15.2,11.7$, and $7.3 \%$, respectively, \\ for all eyelid malignancies. Rates of \\ sebaceous gland carcinoma recurrence, \\ metastasis, and mortality were significantly \\ higher $(P<0.05)$ than those of basal cell \\ carcinoma. The mean interval of recurrence or \\ metastasis after primary treatment was 26.3 \\ months (range 4-112) for all eyelid \\ cancers. \\ Conclusions Although basal cell carcinoma is \\ the most common eyelid cancer in Taiwan, \\ sebaceous gland carcinoma is also common. Of \\ the two, basal cell carcinoma has a better \\ prognosis and sebaceous gland carcinoma has \\ a higher mortality and therefore should be \\ treated much more aggressively. Long-term \\ follow-up is needed after treatment of \\ malignant eyelid tumours. \\ Eye (2003) 17, 216-220. doi:10.1038/ \\ sj.eye. 6700231
}

${ }^{1}$ Department of Ophthalmology Far Eastern Memorial Hospital

Taipei, Taiwan

${ }^{2}$ Department of Ophthalmology National Taiwan University Hospital

Taipei, Taiwan

${ }^{3}$ Chu-Miao Eye Clinic Hsin-Chu, Taiwan

Correspondence:

Dr S-L Liao

Department of

Ophthalmology

National Taiwan University Hospital

7, Chung Shan South Road

Taipei 100, Taiwan

Tel: +886223123456

Fax: +886223934420

E-mail: lang89@

ha.mc.ntu.edu.tw

Received: 19 December 2001

Accepted: 11 April 2002
J-K Wang ${ }^{1}$, S-L Liao ${ }^{2}$, J-R Jou², P-C Lai , SCS Kao ${ }^{3}, \mathrm{P}-\mathrm{K} \mathrm{Hou}{ }^{2}$ and M-S Chen ${ }^{2}$

Keywords: malignant eyelid tumour; basal cell carcinoma; sebaceous gland carcinoma; squamous cell carcinoma; eyelid cancer

\section{Introduction}

Eyelid cancers mainly include basal cell carcinoma (BCC), squamous cell carcinoma (SCC), malignant melanoma (MM), and sebaceous gland carcinoma (SGC). BCC is the most common malignant eyelid tumour. ${ }^{1-7}$ However, SGC occurs much less frequently in the West than it does in China. ${ }^{7}$ The lethality of SGC is also very different from that of BCC. In the present study, we reviewed clinical findings in patients with eyelid cancers at both the National Taiwan University Hospital and Far Eastern Memorial Hospital in Taiwan. In addition, we compared the results of our study with those of various reports about the occurrence of malignant eyelid tumours in other parts of the world.

\section{Materials and methods}

A retrospective analysis was conducted of all malignant tumours of the eyelid at two hospitals in Taiwan. From 1980 to 2000, 124 and three patients with malignant eyelid tumours were treated at the National Taiwan University and Far Eastern Memorial Hospitals, respectively. Tumour locations, histological results, clinical diagnosis, treatment modalities, and follow-up data were collected retrospectively. The mean follow-up period was 62.4 months (range 3-240) for 113 patients. The other 14 patients were not followed up. The statistical analysis was based on a generalized Wilcoxon method and the actuarial method.

\section{Results}

During the 20-year interval, a total of 127 lesions with histopathologic confirmation were 
Table 1 Locations of malignant eyelid lesions

\begin{tabular}{lccccc}
\hline & $\begin{array}{c}\text { Upper lid } \\
(\%)\end{array}$ & $\begin{array}{c}\text { Lower lid } \\
(\%)\end{array}$ & $\begin{array}{c}\text { Inner canthus } \\
(\%)\end{array}$ & $\begin{array}{c}\text { Outer canthus } \\
(\%)\end{array}$ & $\begin{array}{c}\text { More than one region } \\
(\%)\end{array}$ \\
\hline BCC $(n=79)$ & 19.0 & 44.3 & 13.9 & 11.4 & 11.4 \\
SGC $(n=30)$ & 56.7 & 26.7 & 0 & 3.3 & 13.3 \\
Total $(n=127)$ & 33.9 & 37.0 & 9.4 & 8.7 & 11.0 \\
\hline
\end{tabular}

Table 2 Primary treatment modalities

\begin{tabular}{lcccccc}
\hline Treatment modalities & BCC & SGC & SCC & MM & Other cancers & Total \\
\hline Excision with direct closure & 41 & 9 & 5 & 3 & 2 & 60 \\
Excision with eyelid reconstruction & 35 & 15 & 6 & 1 & 0 & 57 \\
Exenteration & 3 & 6 & 0 & 1 & 0 & 10 \\
Surgery with adjuvant radiotherapy & 4 & 4 & 0 & 1 & 0 & 9 \\
\hline
\end{tabular}

included in our study. There were 79 BCCs $(62.2 \%), 30$

SGCs $(23.6 \%), 11$ SCCs $(8.7 \%)$, five MMs (3.9\%), one Kaposi's sarcoma $(0.8 \%)$, and one metastatic cancer from breast $(0.8 \%)$.

A total of 58 cases $(45.7 \%)$ occurred in men, and 69 (54.3\%) occurred in women. There was a slight predominance of BCC in men (43 males, 36 females). However, more lesions of SGC were noted in women (21 females, nine males).

The mean age at diagnosis was 62.6 years (range 10-91) for all patients, 61.8 years (range 10-86) for BCC, and 68.1 years (range 48-91) for SGC. The median age of all eyelid malignancies was 63 years.

All the malignant lesions were more common in the lower $(37.0 \%)$ than the upper eyelid $(33.9 \%)$. BCCs were found most commonly in the lower eyelid (44.3\%). In contrast, most SGCs were seen in the upper eyelid (56.7\%; Table 1).

Of the 127 lesions pathologically confirmed as malignancy, 115 (90.5\%) were clinically diagnosed as malignancy or 'suspected malignancy'. The accuracy in predicting BCC $(72 / 79,91.1 \%)$ was higher than that for SGC $(26 / 30,86.6 \%)$. The accuracy of clinical diagnosis in predicting the correct malignancy was only $100 / 127$ $(78.7 \%)$.

For all malignancies of the eyelid, the most common primary treatment modality was surgical excision with direct closure $(60 / 127,47.2 \%)$, followed by excision with eyelid reconstruction $(57 / 127,44.9 \%)$, and exenteration $(10 / 127,7.9 \%)$. Nine cases received radiotherapy after surgical treatment. The exenteration rate was significantly higher for SGC $(6 / 30,20 \%)$ than that for BCC $(3 / 79,3.8 \%$; Table 2$)$.

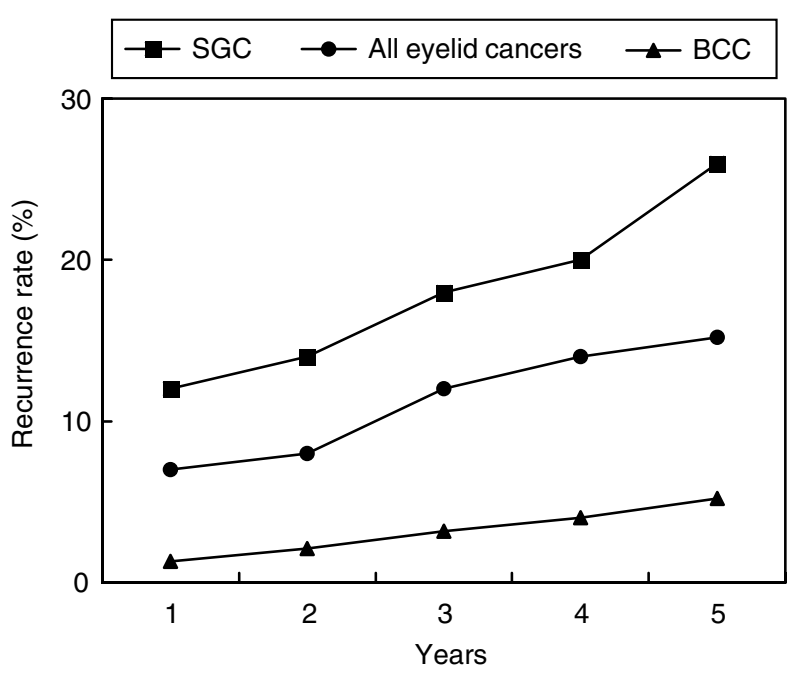

Figure 1 Recurrence rate of eyelid cancers.

A total of 19 patients (16.8\%) had less than 1 year of follow-up, and 48 patients $(42.5 \%)$ had more than 5 years of follow-up. A total of 13 cases (11.5\%) recurred after the primary treatment. Three of them refused to be treated again. The other 10 patients received the second treatment: seven by wide excision, two by exenteration, and one by radiotherapy. Two patients had a second recurrence and one had metastasis. Figure 1 details the local recurrence rate of the eyelid cancers by actuarial analysis. The recurrence rate at 5 years was $5.2 \%$ for BCC. A somewhat higher rate was obtained in patients with SGC: $26 \%$ at 5 years $(P=0.01)$. All patients with eyelid cancers had a 5-year recurrence rate of $15.2 \%$. 
Nine patients had regional lymph node involvement and distant metastasis. Four of them refused further treatment. The other five cases were treated with parotidectomy, radical neck dissection, and adjunctive

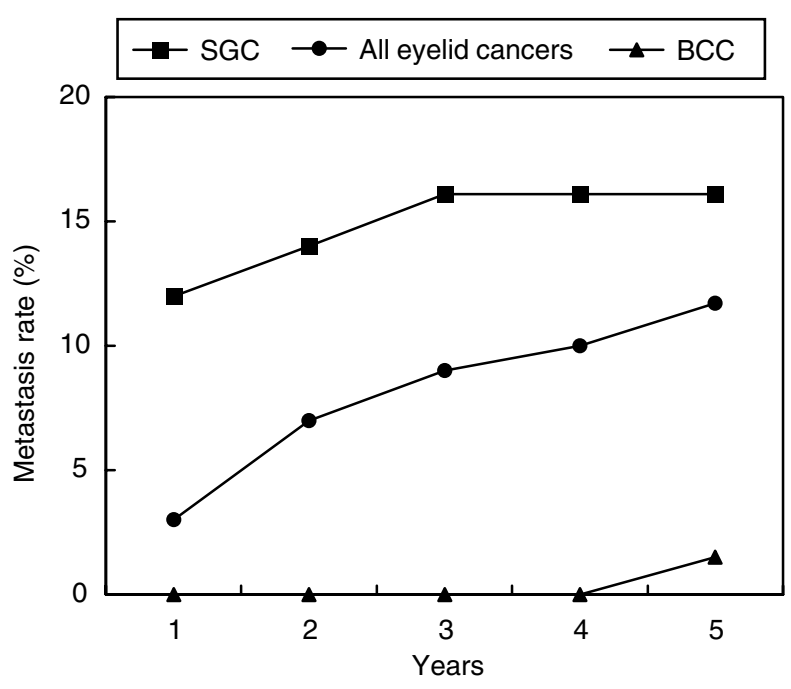

Figure 2 Metastasis rate of eyelid cancers.

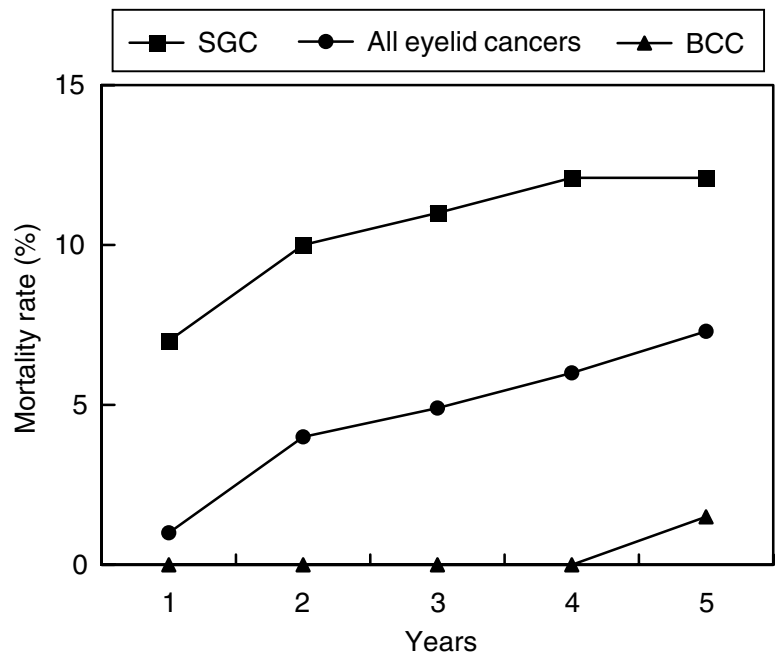

Figure 3 Mortality rate of eyelid cancers. radiotherapy or chemotherapy. Figure 2 shows the actuarial curve of metastasis rate. In $11.7 \%$ of all eyelid cancers, $1.5 \%$ of BCC, and $16.1 \%$ of SGC, tumours metastasized at 5 years. The 5-year metastasis rate was significantly higher for SGC than that for BCC $(P=0.006)$. The mean interval of recurrence or metastasis after primary treatment was 26.3 months (range 4-112) for all eyelid cancers, 47.7 months for BCC (range 5-112), and 17.5 months for SGC (range 4-50).

Seven patients died from the malignancy as a result of metastasis to lungs, liver, or brain. The 5-year mortality rate was $7.3 \%$ for all eyelid cancers, $1.5 \%$ for BCC, and $12.1 \%$ for SGC. There was a significant difference between the 5-year mortality rate of BCC and SGC $(P=0.008)$. Detailed data concerning the mortality rate are presented in Figure 3.

\section{Discussion}

BCC is the most common eyelid malignancy worldwide. It accounts for $80-90 \%$ of all eyelid cancers in Western literature. ${ }^{4}$ However, in various studies of Chinese patients, BCC comprises 28.2-82.8\% of the cases (Table 3). In our study, BCC was the most frequently encountered eyelid cancer $(62.2 \%)$.

SGC is rare among white people, accounting for $1-5.5 \%$ of all eyelid malignancies, far behind BCC and SCC. ${ }^{6-8}$ SGC appears to be much more common in the Chinese population, ranking second, but close, to BCC. Genetics and racial predisposition may play a role. ${ }^{7}$ This greater prevalence in the Chinese population did not appear to result from selective geographic referral patterns, because the studies in Taiwan, Singapore, and Shanghai had similar distributions. ${ }^{3,5,7}$ SGC ranked second in this study, constituting $23.6 \%$ of all eyelid cancers comparable to that from several studies done in Chinese patients. SCC comprised $8.7 \%$ of all malignant lesions of the eyelid, ranking third in this series.

The proportion of pathological diagnoses reported in India is unique though similar; the three most common cancers were SGC $(32.6 \%)$, BCC $(29.8 \%)$, and SCC $(28.1 \%) .{ }^{9}$ In one study in Japan, ${ }^{10}$ SCC, the most common

Table 3 Histologies of eyelid cancers seen in various studies of Chinese patients

\begin{tabular}{lcccc}
\hline & $\begin{array}{c}\text { Our study (Taiwan) } \\
(\mathrm{n}=127) \\
(\%)\end{array}$ & $\begin{array}{c}\text { Hou and Tsai } \\
(\mathrm{n}=39) \\
(\%)\end{array}$ & $\begin{array}{c}\text { Ni et al }^{7} \text { (Shanghai) } \\
(\mathrm{n}=512) \\
(\%)\end{array}$ & $\begin{array}{c}\text { Lee et al }^{5} \text { (Singapore) } \\
(\mathrm{n}=296) \\
(\%)\end{array}$ \\
\hline BCC & 62.2 & 28.2 & 47 & 82.8 \\
SGC & 23.6 & 25.6 & 32.7 & 10.8 \\
SCC & 8.7 & 23.1 & 10 & 3.6 \\
MM & 3.9 & 7.7 & 7.6 & 1.4 \\
Other eyelid cancers & 1.6 & 15.4 & 2.4 & 1.4 \\
\hline
\end{tabular}


Table 4 Histologies of eyelid cancers seen in various studies in the world

\begin{tabular}{|c|c|c|c|c|c|}
\hline & $\begin{array}{l}\text { Our study (Taiwan) } \\
(\mathrm{n}=127) \\
(\%)\end{array}$ & $\begin{array}{c}\text { Ni et al }{ }^{7} \text { (US) } \\
(\mathrm{n}=1543) \\
(\%)\end{array}$ & $\begin{array}{l}\text { Weiner et } a^{6} \text { (Australia) } \\
\qquad(\mathrm{n}=475) \\
(\%)\end{array}$ & $\begin{array}{c}\text { Sihota et al }{ }^{9} \text { (India) } \\
(\mathrm{n}=178) \\
(\%)\end{array}$ & $\begin{array}{c}\text { Abe et al }{ }^{10} \text { (Japan) } \\
(\mathrm{n}=52) \\
(\%)\end{array}$ \\
\hline BCC & 62.2 & 92.5 & 88.8 & 29.8 & 32.6 \\
\hline SGC & 23.6 & 1.5 & 3.8 & 32.6 & 13.5 \\
\hline SCC & 8.7 & 4.6 & 7.4 & 28.1 & 48.1 \\
\hline $\mathrm{MM}$ & 3.9 & 1.1 & 0 & 2.0 & 3.8 \\
\hline Other eyelid cancers & 1.6 & 0.3 & 0 & 7.5 & 2.0 \\
\hline
\end{tabular}

eyelid cancer, was $48.1 \%$, followed by BCC (32.6\%) and SGC (13.5\%; Table 4).

SGC occurs more commonly in women. Among the large studies, between 57 and $77 \%$ of afflicted patients were women. ${ }^{12}$ Of the patients with SGC $70 \%$ were female in this series. The gender distribution of BCC varies. Some report no gender predilection, ${ }^{11}$ and some report that females are more prone to BCC than males. ${ }^{12,13}$ However, in this study, the male-to-female ratio was 1.2. This result was similar to one previous study. ${ }^{14}$

The median age at diagnosis of all eyelid malignancies was 63 years, similar to that found in studies in Singapore, ${ }^{5}$ but lower than that seen in Japan, ${ }^{10}$ where a median age in the 70s was reported. The average age at diagnosis of BCC of the eyelid is nearly $60,{ }^{4}$ which is comparable to the 61.8 years that we report. Patients with SGC are usually between their fifth and ninth decades of life. ${ }^{8}$ In our study, the mean age of patients with SGC was 68.1 years (range 48-91). It was higher than that seen in the Shanghai study, which reported a mean age of 57 years. ${ }^{7}$

All the malignant lesions were more common in the lower than the upper eyelid. This result was the same as that of previous studies. ${ }^{1,2}$ The predominance of BCC in the lower eyelid was shown in various studies ${ }^{2,4,12,13,15}$ as well as in our study. However, more SGCs occurred in the upper eyelid in various studies ${ }^{7,8,16,17}$ and in this study. In most series, the upper eyelid is involved two to three times more frequently than the lower lid for SGC. This has been attributed to the greater number of meibomian glands in the upper lid. ${ }^{8}$ In this series, the upper lid was involved 2.1 times more frequently than the lower lid for SGC.

In examining an actual malignant lesion, it is more important for the clinician to suspect malignancy than to identify the particular type of malignancy. This is because a patient with a malignant lesion can only be harmed if the clinician suspects a benign lesion and elects not to remove it. ${ }^{2}$ In this study, the clinical accuracy in predicting eyelid malignancy was $90.5 \%$, and is comparable with that in the study of Tesluk. ${ }^{2}$ The accuracy in predicting BCC was higher than that for SGC. The possible reason might be that SGC can masquerade as chronic blepharoconjunctivitis or chalazion.

The recurrence rate of $\mathrm{BCC}$ at 5 years was $5.2 \%$ in this series. The mean time to recurrence or metastasis was nearly 4 years for BCC. One case of BCC recurred after about 10 years. In previous studies of eyelid BCC, a recurrence rate of $5.36-6.1 \%$ was observed over 3 or 4 more years of follow-up. ${ }^{12,13}$ One of the cases with BCC had orbital invasion after exenteration and removal of orbital bone. After 5 years, the patient received radiotherapy as a result of regional lymph node metastasis, but still died from lung metastasis. This patient was the only one who had metastasis and died from BCC. The 5-year mortality rate was $1.5 \%$. Mortality from eyelid and medial canthal BCC ranged from 2 to $11 \%$, based on data from several decades ago. ${ }^{4}$

The local recurrence rate of $26 \%$ was observed at 5 years for SGC. The mean interval to recurrence or metastasis was about 1.5 years, mostly within 4 years. Sebaceous carcinoma is reported to recur in 6-29\% of the cases. The majority of all recurrences appear within the first 4 years after treatment. ${ }^{18}$ The rate of metastasis at 5 years was $16.1 \%$. Four of our patients with SGC developed regional lymph node metastasis and received parotidectomy, radical neck dissection, and chemotherapy or radiotherapy. Three of them died from brain or liver metastasis. The mortality rate at 5 years was $12.1 \%$. According to the literature, distant metastasis affects $14-25 \%$ of the cases and involves lymph node or hematogenous spread into liver, lungs, brain, and bones. ${ }^{18}$ SGC is traditionally considered among the most lethal of all tumours of the ocular adnexa. Mortality from SGC has been estimated to be from 6 to $30 \%$ in a previous study. 8

The mean time to recurrence or metastasis after primary treatment was longer for BCC than that for SGC, implying that BCC must be followed up for a longer period. As a result of its highly invasive activity, SGC had higher recurrence, metastasis, and mortality rates than did BCC $(P<0.05)$. 
In summary, malignant eyelid tumours in Taiwan develop predominantly in the elderly on the lower lids. The majority of eyelid cancers in this study were BCCs, for which a long-term follow-up is required, but more importantly, a high occurrence rate of SGCs is uniquely evident in Taiwan. In the light of SGCs being associated with an aggressive clinical course and poor prognosis, this tumour should be treated aggressively.

\section{References}

1 Leventhal HH, Messer RJ. Malignant tumours of the eyelid. Am J Surg 1972; 124: 522-526.

2 Tesluk GC. Eyelid lesions: incidence and comparison of benign and malignant lesions. Ann Ophthalmol 1985; 17: 704-707.

3 Hou PK, Tsai YS. Eyelid tumour: a statistical study. Acta Soc Ophthalmol Sin 1977; 16: 34-36.

4 Margo CE, Waltz K. Basal cell carcinoma of the eyelid and periocular skin. Surv Ophthalmol 1993; 38: 169-192.

5 Lee SB, Saw SM, Eong KGA et al. Incidence of eyelid cancers in Singapore from 1968 to 1995. Br J Ophthalmol 1999; 83: 595-597.

6 Weiner JM, Henderson PN, Roche J. Metastatic eyelid carcinoma. Am J Ophthalmol 1986; 101: 252-254.

7 Ni C, Searl SS, Kuo PK et al. Sebaceous cell carcinomas of the ocular adnexa. In: NiC, Albert DM (eds). Tumours of the Eyelid and Orbit: a Chinese-American Collaborative Study. Int Ophthalmol Clin 1982; 22: 23-61.
8 Kass LG, Hornblass A. Sebaceous carcinoma of the ocular adnexa. Surv Ophthalmol 1989; 33: 477-490.

9 Sihota R, Tandon K, Betharia SM et al. Malignant eyelid tumours in an Indian population. Arch Ophthalmol 1996; 114: 108-109.

10 Abe M, Ohnishi Y, Hara Y et al. Malignant tumour of the eyelid: clinical survey during a 22-year period. Jpn J Ophthalmol 1983; 27: 175-184.

11 Payne JW, Duke JR, Butner R et al. Basal cell carcinoma of the eyelids. Arch Ophthalmol 1969; 81: 553-558.

12 Pieh S, Kuchar A, Novak P et al. Long term results after surgical basal cell carcinoma excision in the eyelid region. Br J Ophthalmol 1999; 83: 85-88.

13 Steinkogler FJ, Scholda CD. The necessity of long-term follow up after surgery for basal cell carcinomas of the eyelid. Ophthalmic Surg 1993; 24: 755-758.

14 Fitzpatrick PJ, Thompson GA, Easterbrook WM et al. Basal and squamous cell carcinoma of the eyelids and their treatment by radiotherapy. Int J Radiat Oncol Biol Phys 1984; 10: $449-454$.

15 Cook BE, Bartley GB. Epidemiologic characteristics and clinical course of patients with malignant eyelid tumours in an incidence cohort in Olmsted County, Minnesota. Ophthalmology 1999; 106: 746-750.

16 Doxanas MT, Green WR. Sebaceous gland carcinoma: review of 40 cases. Arch Ophthalmol 1984; 102: 245-249.

17 Rao NA, Hidayat AA, McLean JW et al. Sebaceous carcinoma of the ocular adnexa: a clinicopathologic study of 104 cases, with five-year follow-up data. Hum Pathol 1982; 13: 113-122.

18 Zurcher M, Hintschich CR, Garner A et al. Sebaceous carcinoma of the eyelid: a cliniopathological study. $\mathrm{Br} \mathrm{J}$ Ophthalmol 1998; 82: 1049-1055. 